\title{
A Comparative Analysis and Experimental Study on Wireless Aerial and Underwater Acoustic Communications
}

\author{
Raja Jurdak*, Cristina Videira Lopes*, Pedro M. Q. Aguiar ${ }^{\dagger}$, Pierre Baldi* \\ * School of Information and Computer Science, University of California, Irvine CA 92697 \\ California Institute for Telecommunications and Information Technology Calit2 \\ \{rjurdak, lopes, pfbaldi\} @ics.uci.edu \\ †Institute for Systems and Robotics / IST, Lisboa,Portugal \\ aguiar@isr.ist.utl.pt
}

\begin{abstract}
Widely available speakers and microphones in electronic devices motivate the use of generic audio hardware to establish acoustic communication links. Acoustic communication with generic hardware requires software modems for data modulation and demodulation. The resulting acoustic communications system paves the way for cheap and easily deployable underwater acoustic sensor networks through the use of audio hardware built in to the sensor modules. In this paper, we explore the potential of the acoustic communication system for both aerial and underwater environments. Our experiments profile both media with generic microphones and speakers. An initial test of the channel's underwater data communication capability has revealed that the channel can transfer data at $12 \mathrm{bps}$ and $24 \mathrm{bps}$, with respective error rates of $10 \%$ and $20 \%$, up to a distance of $10 \mathrm{~m}$.
\end{abstract}

\section{INTRODUCTION}

Recent increases in the processing power and in the availability of speakers and microphones in embedded and mobile devices have fueled interest in designing software acoustic modems for audio communications. Many current electronic devices already includes audio speakers and microphones, which can support a data communications through a low bit rate modulated audio signal. Supporting low bit rate audio wireless communications through the usable bandwidth of existing acoustic hardware enables cheap and easily deployable solutions for several emerging applications, such as ubiquitous computing and sensor networks.

Reliable communications through audio signals are already established for certain applications. Telephone modems have for decades used acoustic signals over copper cables to modulate information, reaching a maximal data rate of $56 \mathrm{Kbps}$. Acoustic signals have also been the top choice for wireless underwater communications, with applications that include oil prospecting, marine biology research, and environmental monitoring.

Most existing wired and wireless audio communication techniques rely on specialized hardware. In particular, acoustic communication typically requires hardware that performs sig- nal modulation and transmission at the sender side, in addition to hardware capable of receiving and decoding the audio signal at the receiver side. In the case of wired acoustic communications, telephone modems typically integrate all the hardware required to modulate, transmit, receive and demodulate audio signals. Wireless underwater acoustic communications often rely on specialized and expensive acoustic modems, as well as acoustic transducers and hydrophones to send and receive the signals underwater. Requiring the use of specialized hardware limits the pervasive use of acoustic communications, since it typically reduces system portability, increases the system cost, and requires more time and effort for installing and interfacing hardware components

In the past, low processing speeds dictated the use of specialized hardware for acoustic modulation. An alternative approach which overcomes the compatibility, cost, and portability drawbacks of hardware modems is the implementation of acoustic modulation and demodulation in software, as suggested in [1] for wireless aerial acoustic communications. Recent advances in miniaturization and circuit integration have yielded smaller and more powerful processors that are capable of efficiently running acoustic modulation and demodulation software. The transmission and reception of the software modulated acoustic signal can also avoid using specialized hardware through generic speakers and microphones.

Eliminating the need for specialized hardware for acoustic communication greatly reduces the system cost, enabling the networking of any devices with speakers and microphones. Within this context, our study here is part of a project to deploy a short range shallow water network to monitor pollution indicators in Newport Bay, CA [2] and to provide the data to environmental engineers in near real time. We expect the network to consist of general purpose sensor modules that use software modems and generic built-in hardware to communicate acoustically and send the data to the base station.

For our application, we have selected mote-class computers, which are powerful enough to perform the limited in-network 
processing and are affordable enough to enable the deployment of a dense network at reasonable cost. In particular, we have selected the Tmote Invent module, from Moteiv Corp. [3], which has an on-board SSM2167 microphone from Analog Devices sensitive to $100 \mathrm{~Hz}$ to $20 \mathrm{kHz}$, and an on-board TPA0233 speaker amplifier from TI with an $8 \mathrm{ohm}$ speaker that has a range of $400 \mathrm{~Hz}$ to $20 \mathrm{kHz}$. We intend to exploit the on-board microphone and speaker to establish short range acoustic links among Invent modules.

In this paper, we explore the fundamentals of aerial and underwater acoustics in order to profile these channels and to design software acoustic modems that can run on a general purpose mote modules, such as the Tmote Invent unit. We also investigate the acoustic communication capability of the system consisting of the software modems and the generic speakers and microphones in underwater experiments. The experiments reveal that the aerial channel favors frequencies above $1 \mathrm{Khz}$ because of high ambient noise at low frequencies. In contrast, the underwater channel favors lower frequencies as a result of the vinyl membrane that we use for speaker waterproofing which seems to resonate better at lower frequencies.

The remainder of this paper is organized as follows. Section II discusses the related work on both hardware and software acoustic modems in aerial and underwater environments. Section III presents the basic principles and relationships governing acoustic signal propagation in both air and water. Section IV addresses our performance evaluation of generic acoustic hardware for profiling the aerial and underwater media. We use an FSK modem to evaluate the data transfer characteristics of our acoustic underwater communication system. Finally, section V concludes the paper

\section{Acoustic Communications And Modems}

Many applications can benefit from acoustic communications, so several works have considered the modulation of sound in order to transmit information. These works can be broadly classified into 2 categories: (A) hardware modems; and (B) software modems.

\section{A. Hardware Modems}

Earlier efforts in acoustic communication have focused on using specialized and dedicated hardware for sound modulation and demodulation. There are 2 main applications for hardware acoustic modems: (1) phone line modems; and (2) underwater acoustic modems.

1) Phone line modems: Modems originally allowed longdistance point-to-point communication using the voice band in ordinary telephone networks. The first modems were acoustically coupled: a user placed the telephone receiver into a modem handset and the modem sent tones to the telephone. Early acoustic modems transmitted at 300 bits per second. Direct-connect modems, which interface directly with the telephone line, have replaced these acoustic modems. They are less bulky, give a better connection, and avoid the background noise problems of acoustic modems. Modern telephone line modems transmit at bit rates of up to $56 \mathrm{Kbps}$.
2) Underwater Acoustic Modems: Acoustic underwater communication is a mature field and there are several commercially available underwater acoustic modems $[4,5]$. The prohibitive cost of commercial underwater modems has been an obstacle to the wide deployment of dense underwater networks, until the recent development of research versions of hardware acoustic modems.

Both of the efforts reported in [6] and [7] aim at making underwater acoustic modems more affordable and accessible to the research community by developing specialized affordable hardware. Our work aims at driving the cost even lower and at making acoustic underwater communications even more accessible through the development of software acoustic modems that can operate on generic hardware platforms.

The work in [8] uses generic microphones and speakers along with a specialized integrated circuit that generates and ASK or FSK modulated sound signal in order to demonstrate the acoustic communication capability underwater. Vasilescu et al. achieve a bit rate in the order of tens of bits per second up to about 10 to 15 meters. Our work resembles their work in the use of generic microphones and speakers for acoustic communications, but our work differs in its implementation of software modems with a generic platform rather than a specialized integrated circuit.

\section{B. Software Modems}

With the rapid increase in processor speeds, the idea of implementing acoustic modems in software became feasible. The cost of software acoustic modems is limited to the development cost, after which the per unit cost is zero. Because of these attractive features, researchers have started exploring software acoustic modems for aerial acoustic communications and underwater acoustic communications. Lopes and Aguiar have investigated using software modems for aerial acoustic communications [1] in ubiquitous computing applications. Many ubiquitous computing applications can make use of generic microphones and speakers available on computing devices to communicate through sound, which has some attractive properties for indoor wireless communication. First, unlike radio waves sound rarely penetrates room walls, which reduces interference and promotes privacy. Second, unlike infrared sound does not require a line-of-sight to achieve communication, which is desirable in a indoor environment with many obstacles causing severe multi-path effects. The drawback of using sound for aerial communications is the low bit rate (the work in [1] reports a bit rate in the order of tens of bits per second). However, this low bit rate is quite suitable for many ubicomp applications. Underwater sensor networks can also build on and benefit from low bit rate software acoustic modems, which is why our study here explores the communication potential of generic hardware coupled with software modems in both aerial and underwater environments. 


\section{Fundamentals of ACOUstics}

\section{A. Aerial Acoustics}

The signal-to-noise ratio (SNR) quality metric at the receiver of an emitted aerial acoustic signal is governed by the following equation:

$$
S N R_{a}=S L_{a}-T L_{a}-N L_{a}
$$

where $S L_{a}$ is the source level, $T L_{a}$ is the transmission loss, $N L_{a}$ is the noise level. All quantities in Equation 1 are in $d B$ relative to the power density level of the threshold of human hearing, which is $10^{-12} \mathrm{~W}$ atts $/ \mathrm{m}^{2}$.

1) Source Level: Typically, the specifications of audio speakers indicate the speaker's maximum emitted signal power. To obtain the source level $S L_{a}$ of the speaker, we first obtain the signal intensity at $1 \mathrm{~m}$ from the speaker, assuming that the signal spreading is cylindrical with a unity radius within a distance of $1 \mathrm{~m}$ from the source:

$$
I_{t}=\frac{P_{t}}{2 \times \pi \times 1^{2}}
$$

where $I_{t}$ is expressed in $W$ atts $/ \mathrm{m}^{2}$, and $P_{t}$ is the transmission power in Watts. The following equation determines the source level $S L_{a}$ relative to the threshold level of human hearing:

$$
S L_{a}=10 \log \left(\frac{I_{t}}{10^{-12}}\right)
$$

2) Transmission Loss: The transmission loss of the aerial acoustic signal depends on the surroundings. For example, in an indoor environment, the signal reflects on objects and walls, and the signal is approximated to spread in a cylinder between source and receiver. In practical terms, a smaller portion of the acoustic signal is absorbed by the surroundings inside a room than outdoors. It is particularly for this reason that the same sound or music heard indoors seems louder than it does outdoors.

The signal transmission loss in an indoor environment is expressed as [9]:

$$
T L_{a}=10 \log (d)
$$

where $d$ is the distance in meters between the sender and receiver, and $T L_{a}$ is expressed in $\mathrm{dB}$.

3) Noise Level: The noise level for aerial indoor acoustic environments results from many factors, including movement of people and objects, vibration and sounds from machines, and occasional noise impulses from the surroundings such as cell phones. In typical indoor environments, the noise sources typically add to the background noise at lower frequencies, suggesting a higher noise level for these frequencies.

\section{B. Underwater Acoustics}

1) The Passive Sonar Equation: The passive sonar equation [10] characterizes the signal to noise ratio $\left(S N R_{u}\right)$ of an emitted underwater signal at the receiver:

$$
S N R_{u}=S L-T L_{u}-N L_{u}+D I
$$

where $S L$ is the source level, $T L_{u}$ is the underwater transmission loss, $N L_{u}$ is the noise level, and $D I$ is the directivity index. All the quantities in Equation 5 are typically in $d B$ re $\mu \mathrm{Pa}$, where the reference value ${ }^{1}$ of $1 \mu \mathrm{Pa}$ amounts to $0.67 \times 10^{-22} \mathrm{Watts} / \mathrm{cm}^{2}$ [10]. However, we will use the same reference for the underwater signal as for the aerial acoustic signal (i.e. the threshold of human hearing at $10^{-12} \mathrm{Watts} / \mathrm{m}^{2}$ ) in order to compare results more effectively. In the rest of the paper, we use the shorthand notation of $d B$ to signify $d B$ re $10^{-12}$, unless otherwise mentioned.

The directivity index $D I$ for our network is zero because we assume omnidirectional hydrophones. Although using a directive hydrophone, as described in [12], reduces power consumption, for simplicity we assume omnidirectional hydrophones, thus $D I=0$ in our case.

2) Transmission Loss: The transmitted signal pattern has been modelled in various ways, ranging from a cylindrical pattern to a spherical one. The following expression governs acoustic signals propagation in shallow water [10]:

$$
T L_{u}=10 \times \mu \log d+\alpha d \times 10^{-3}
$$

where $d$ is the distance between source and receiver in meters, $\alpha$ is the frequency dependent medium absorption coefficient in $d B / \mathrm{km}$, and $T L$ is in $d B$. Equation 6 contains two components: a distance dependent attenuation component; and a component representing medium absorption that depends on both distance and frequency. The variable $\mu$ depends on the signal spreading pattern. If the acoustic signal spreads in all directions from the sound source, then $\mu$ is equal to 2 . If the acoustic signal signal spreads in a cylindrical pattern from the source (as is the case for signals propagating along the surface or ocean floor), then $\mu$ equals to 1 , which renders equation 6 identical to equation 4 for indoor aerial acoustic signals. In shallow water cases, the value of $\mu$ lies somewhere between 1 and 2, depending on the depth.

Equation 6 indicates that the transmitted acoustic signal loses energy as it travels through the underwater medium, mainly due to distance dependent attenuation and frequency dependent medium absorbtion [13].

3) Source Level: The transmitter source level (SL) of underwater sound relates to signal intensity $I_{t}$, which in turn depends on the transmission power. Given the transmission power $P_{t}$, the transmitted intensity of an underwater signal at $1 \mathrm{~m}$ from the source can be obtained through the following expression [10]:

$$
I_{t}=\frac{P_{t}}{2 \pi \times 1 m \times H}
$$

in $W$ atts $/ \mathrm{m}^{2}$, where $H$ is the water depth in $\mathrm{m}$. We can then use $I_{t}$ in equation 3 in order to solve for $S L$.

4) Noise Level: Factors contributing to the noise level $N L_{u}$ in shallow water networks include waves, shipping traffic, wind level, biological noise, seaquakes and volcanic activity,

\footnotetext{
${ }^{1}$ The notation $\mathrm{dB}$ re $\mathrm{X}$ indicates the decibel level of a signal relative to a reference value $\mathrm{X}$.
} 


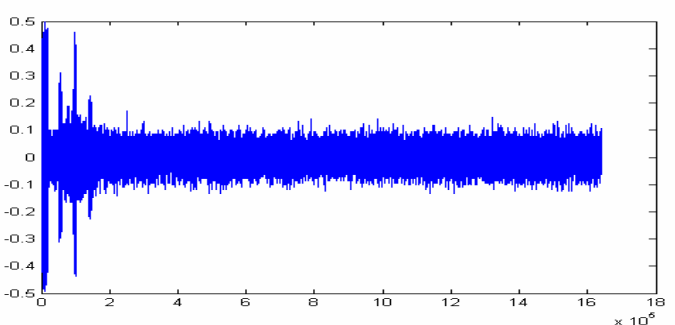

(a)

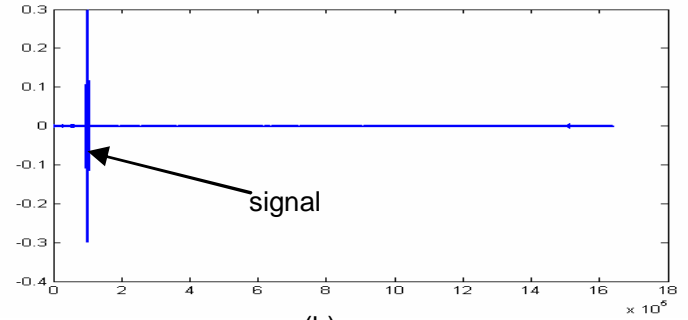

(b)

Fig. 1. (a) Captured signal plot represented in amplitude versus time samples. (b) Filtered version of the captured signal.

and the impact of each of these factors on $N L_{u}$ depends on the particular setting. For instance, shipping activity may dominate noise figures in bays or ports, while water currents are the primary noise source in rivers. In a swimming pool environment, the main sources of underwater noise are swimmers, vibrations from people walking near the pool, and water pumps.

\section{EXPERIMENTS}

\section{A. Aerial Channel Profiling}

The first set of experiments profile the aerial acoustic channel. In these experiments, we use an IPAQ 5550, with a built-in audio speaker, as the transmitter of the acoustic signal. The signal is then captured by a microphone attached to a desktop PC, where the signal is recorded into a file for analysis. We conducted the experiments in a typical office environment with distances ranging from 1 to 7 meters at 2 meter increments.

In an earlier study on aerial acoustic communications [14], we had determined that PC speakers and microphones have the best response at frequencies up to about $7 \mathrm{KHz}$. Building on that result, we have limited our analysis of the aerial channel profile to the frequency range from $400 \mathrm{~Hz}$ to $6700 \mathrm{~Hz}$ at 100 $\mathrm{Hz}$ increments. Each frequency tone is sent for a duration of 1 second, and the full signal consists of a sequence of the signals separated by guard times.

Figure 1(a) shows an example of a captured signal, and Figure 1(b) shows the corresponding filtered signal. To obtain the signal quality of each frequency $f_{i}$ of a signal received from distance $d_{j}$ meters away, we apply a $100 \mathrm{~Hz}$ Equiripple band pass filter centered at $f_{i}$ to the received signal. The filtered signal shape includes the transmitted tone at $f_{i}$, whose average amplitude we indicate as $S$, along with all the background noise within the frequency range $f_{i}-50$ to $f_{i}+50$. The background noise is distinguishable in all the

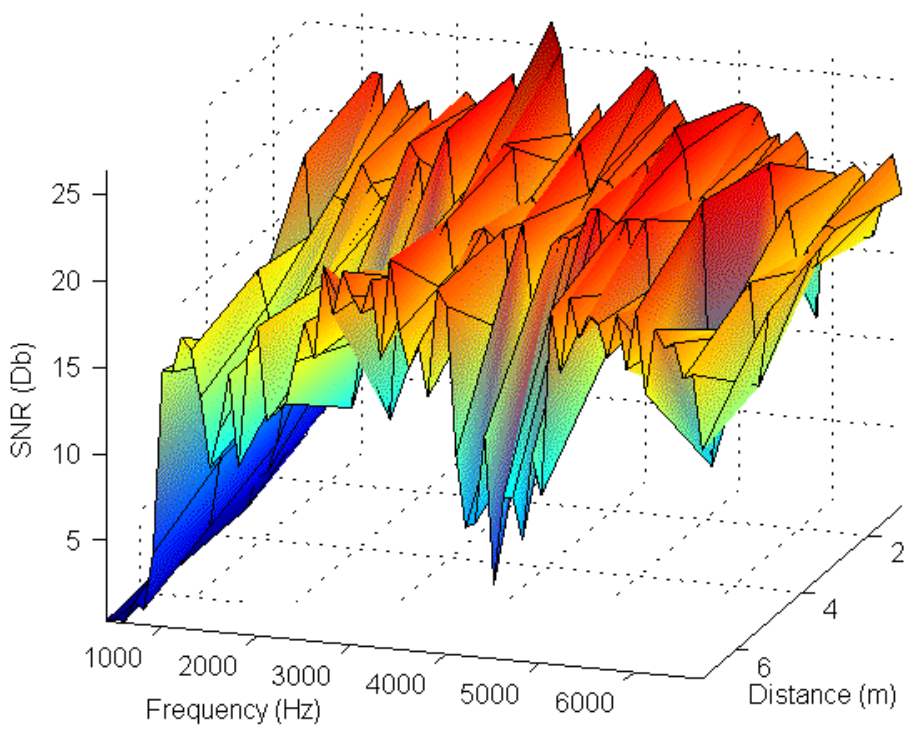

Fig. 2. Received signal quality in the indoor aerial channel

temporal components of the signal during which the tone at $f_{i}$ is not transmitted, and we indicate the average amplitude of the noise as $N$. Through this filtering process, we can obtain the signal to noise ratio $S N R_{a}\left(f_{i}, d_{j}\right)$ of the channel for each frequency $f_{i}$ and distance $d_{j}$ as:

$$
S N R_{a}\left(f_{i}, d_{j}\right)=10 \log \frac{S\left(f_{i}, d_{j}\right)}{N\left(f_{i}, d_{j}\right)}
$$

Figure 2 illustrates the measured $S N R_{a}\left(f_{i}, d_{j}\right)$ for the various frequencies $f_{i}$ and distances $d_{j}$ in our experiments to profile the aerial channel. The most prominent trend in Figure 2 is that the received signal quality for low frequency tones below $1 \mathrm{Khz}$ is considerably lower than for higher frequencies. In fact, the received signal quality gradually increases with increasing frequency from $400 \mathrm{~Hz}$ to $1000 \mathrm{~Hz}$. For frequencies between $1 \mathrm{Khz}$ and $2.5 \mathrm{Khz}$, the signal quality is generally stable with minor variations among frequencies. The signal quality is highest for frequencies above $2.5 \mathrm{Khz}$, which confirms the earlier results in [14]. In regards to distance, the signal quality degrades with a trend following the expected signal attenuation in most cases. The cases in which the received signal quality does not adhere to the attenuation can be attributed to multi-path effects (such as reverberation) and varying exposure to noise sources at the positions within our experiment area.

We can use the measured $S N R_{a}\left(f_{i}, d_{j}\right)$ values to obtain the observed $N L_{a}\left(f_{i}, d_{j}\right)$ values for each frequency $f_{i}$ and distance $d_{j}$ in the indoor aerial acoustic channel. The audio speakers of the IPAQ 5550 have a maximum audio transmission power $P_{t}$ of 2 Watts. Through equations 2 and 3, the corresponding source level $S L_{a}$ of the IPAQ acoustic signal is $10.5029 \mathrm{~dB}$. For the remainder of this analysis, we assume that $S L_{a}$ is uniform at $10.5029 \mathrm{~dB}$ across frequencies in our 


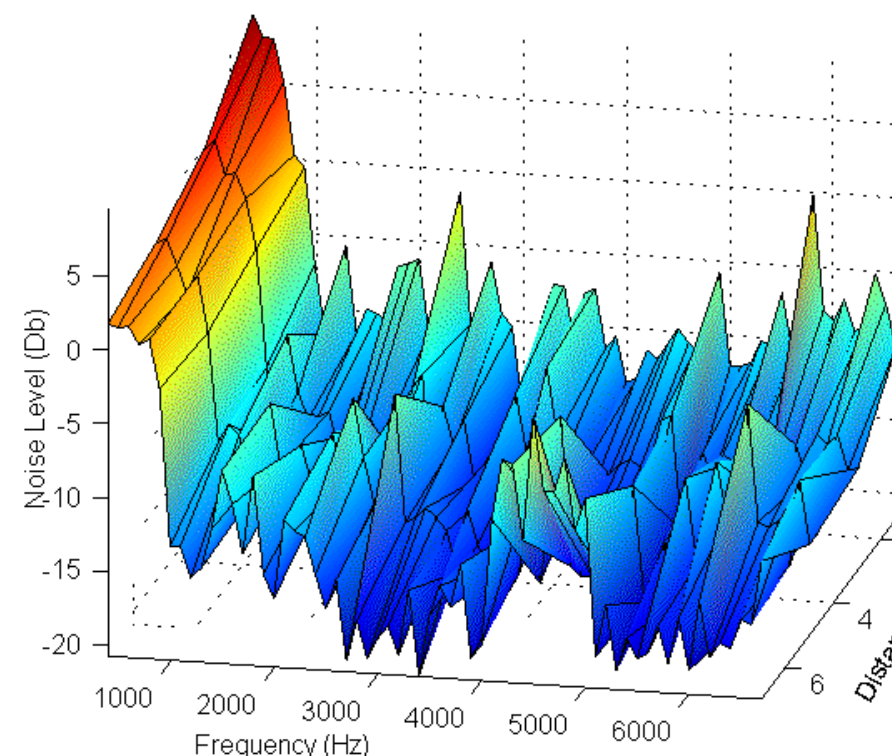

Fig. 3. Apparent noise level at different frequencies and distances in the indoor aerial environment

spectrum from $400 \mathrm{~Hz}$ to $6700 \mathrm{~Hz}^{2}$. Having determined $S L_{a}$ and $S N R_{a}\left(f_{i}, d_{j}\right)$ for each $\left(f_{i}, d_{j}\right)$ pair, we can obtain the transmission loss for $T L_{a}\left(f_{i}, d_{j}\right)$ for each distance $d_{j}$ from equation 4 in order to compute $N L\left(f_{i}, d_{j}\right)$, which is shown in Figure 3.

According to Figure 3, the apparent noise level in the indoor acoustic channel is higher for frequencies between 400 $\mathrm{Hz}$ and $1000 \mathrm{Khz}$. The higher apparent noise level at low frequencies is due to interference from electronic devices, such as computers, monitors, printers in the form of white noise. Also, the movement of people and objects creates transient noise impulses at low frequencies.

For some frequencies in Figure 3, the apparent noise level seems to decrease as the distance between the sender and receiver increases. This is probably explained by the severe multi-path propagation of the acoustic signal indoors which causes a larger portion of the signal to arrive at the receiver than in the cylindrical spreading case. As such, the expression for $T L_{a}$ overestimates the transmission loss. The effect of this overestimation of $T L_{a}$ becomes more apparent at larger distances, causing the model to attribute less of the signal quality degradation to background noise.

\section{B. Underwater Medium Profiling}

The second set of experiments explores the underwater acoustic communication capability of generic desktop PC speakers ${ }^{3}$. We have selected Sony SRS-P7 PC speakers and Labtec PC microphone, both of which are cheap off-theshelf components. In order to waterproof the speakers and

\footnotetext{
${ }^{2}$ We need to make this assumption since the IPAQ specifications do not provide the frequency response of the speakers. In the subsequent discussion, we comment on the effect of this assumption on the results.

${ }^{3}$ The Tmote Invent units were still in production at the time of this study, so we have chosen speakers and microphones of comparable specifications to the the Tmote speakers and microphones.
}

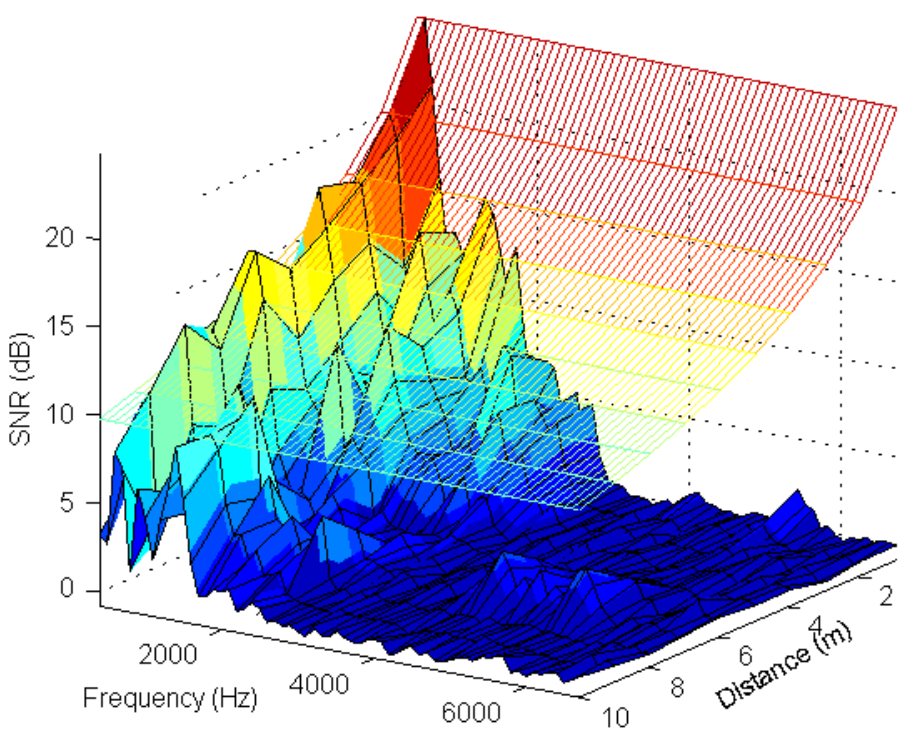

Fig. 4. Profile of the underwater channel: The solid plot represents the measured SNR and the transparent plot represents the expected SNR

microphones and still maintain most of their acoustic properties, we have placed each of them inside vinyl membrane containers. We sealed the vinyl containers around the wires of the components with electric tape to prevent water leakages into the components.

For our experiments, we used two laptops, with one laptop attached to the speakers acting as a sender, and another attached to the microphone acting as a receiver. The microphone and speakers are placed inside a controlled water environment at a depth of about $50 \mathrm{~cm}$, while the wires and the laptops remained outside the water. We conducted two sets of underwater experiments: (1) experiments to profile the medium's frequency response; and (2) experiments to evaluate data transmission capability.

Our application has unique channel characteristics that differ from underwater channels that appear in the related literature $[10,15,16]$. First, our channel is not limited to the underwater medium. Our channel also includes the generic speakers and microphone, whose response and coupling with the underwater environment is unknown. In addition, our channel includes the vinyl membranes which may amplify or attenuate certain frequencies.

We have performed experiments to assess the frequency profile of the channel. As in the aerial acoustic experiments above, we use $S N R_{u}$ as the quality indicator of the received signal, and we profile the medium through the same signal and SNR calculation method. We performed the underwater experiments for distances $d_{j}$ ranging from $1 \mathrm{~m}$ to $10 \mathrm{~m}$ at $1 \mathrm{~m}$ increments. At each distance $d_{j}$, we conducted the measurements three times and obtained the average $S N R_{u}\left(f_{i}, d_{j}\right)$ of the three samples for $f_{i}$.

Figure 4 shows the $S N R_{u}\left(f_{i}, d_{j}\right)$ results for each frequency $f_{i}$ at each distance $d_{j}$. The solid plot in Figure 4 represents the measured SNR. In order to better understand the signal 
interaction, we also computed the expected SNR through the following method. Let the $\left(f_{m}, d_{n}\right)$ be the frequency and distance pair with the highest received SNR. We can use the speaker transmission power $P_{t}$ of 0.8 Watts [11] to obtain the source level $S L$ through equations 7 and 3 . We can also get the transmission loss $T L_{u}\left(f_{m}, d_{n}\right)$ through equation 6 with a value of $\mu$ equals to 1.5 , which is suitable for a shallow water setting [10]. Finally, we can obtain the noise level $N L\left(f_{m}, d_{n}\right)$ through equation 5. To determine the expected SNR, we assume that NL for all frequencies and distances is uniform and equal to $N L\left(f_{m}, d_{n}\right)^{4}$. We can then obtain the expected $S N R\left(f_{i}, d_{j}\right)$ for all frequencies by simply using equation 5. The transparent plot in Figure 4 represents the expected SNR.

It is obvious from the figure that low frequency signals have a similar SNR value as the expected case, whereas there is an increasing gap between the expected and measure SNR values as the frequency increases. Along the distance axis, the SNR of lower frequency signals closely follows the $T L_{u}$ model with a $\mu$ value of 1.5 , whereas this trend also becomes less evident at higher frequencies. To explore these interactions further, we focus our discussion on the measured SNR plot.

The first observation is that for all distances, lower frequencies in general had a higher signal quality than higher frequencies. In particular, $S N R_{u}$ is too low to distinguish the signals from noise for frequencies above $3 \mathrm{Khz}$. Through the analysis in section IV-A, we know that speakers of this size do not exhibit degraded performance with the frequency range from $3 \mathrm{Khz}$ to $7 \mathrm{Khz}$. As such, we can rule out the speaker hardware as a cause for the low quality of the received signal in this frequency range. The two other possible causes for this signal quality degradation are: the presence of higher background noise in the frequency range between 3 and $7 \mathrm{Khz}$; and the poor vibration properties of the encompassing vinyl membrane for these frequencies (yielding a lower $S L$ ).

Another interesting observation is the frequency selectivity of this particular channel. For example, the experiments for most distances yielded a higher $S N R$ for the frequency of $1400 \mathrm{~Hz}$ than at $1100 \mathrm{~Hz}$. This is probably due to the speaker and microphone design, to higher ambient noise at certain frequencies, or to the vinyl membranes, which might resonate at some frequencies better than others.

The above discussion strongly suggests that the vinyl membrane creates unknown frequency-specific variations for $S L$. It also suggests that the noise level $N L$ in our shallow water setting has unknown frequency-specific patterns. We can rewrite equation 5 to place all unknowns on one side of the equation:

$$
S L_{u}\left(f_{i}\right)-N L_{u}\left(f_{i}, d_{j}\right)=S N R_{u}\left(f_{i}, d_{j}\right)+T L_{u}\left(f_{i}, d_{j}\right)
$$

Through equation 9, we can obtain the difference $S L_{u}\left(f_{i}\right)$ $N L_{u}\left(f_{i}, d_{j}\right)$ since we have measured $S N R_{u}\left(f_{i}, d_{j}\right)$ and we

\footnotetext{
${ }^{4}$ Although this assumption may seem simplistic, the purpose is simply estimating the expected trend of the SNR at the receiver. In the subsequent discussion, we drop this assumption and explore the noise level for every frequency and distance.
}

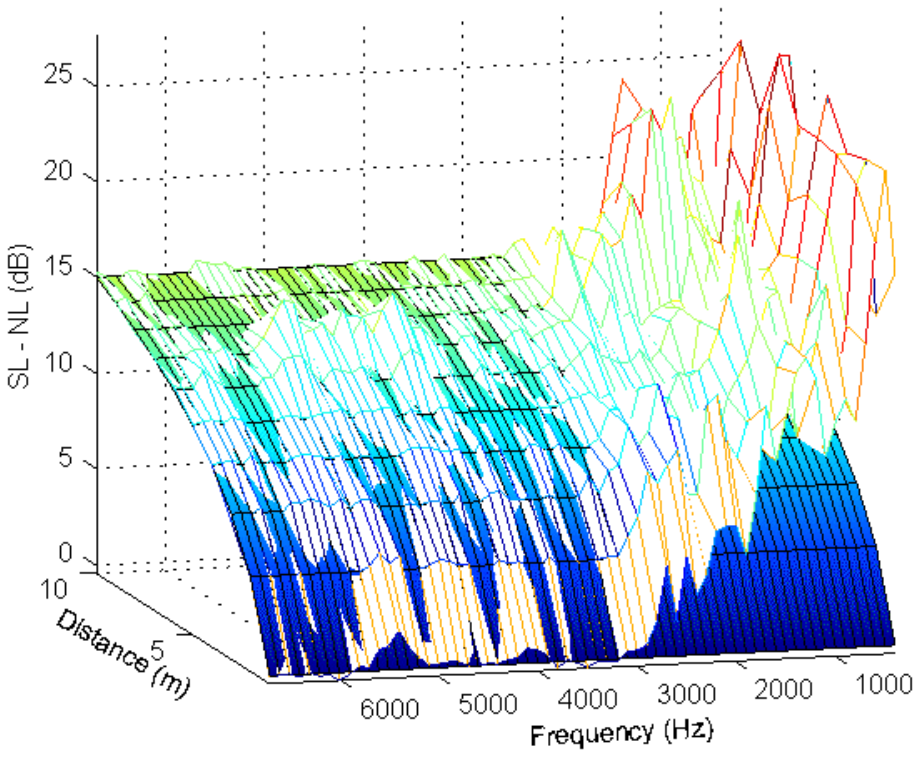

Fig. 5. Difference between source level and noise level for each frequency and distance pair. The solid graph indicates the difference the points at which the difference yields an $S N R$ of $0 \mathrm{~dB}$. The white graph shows the measured values.

can easily compute $T L_{u}\left(f_{i}, d_{j}\right)$ from equation 6.

Figure 5 plots the difference of the two unknowns, $S L$ and $N L$. The solid graph represents the difference between $S L$ and $N L$ for each frequency $f_{i}$ that yields a $0 S N R$ at a receiver $d_{j}$. The white graph shows the difference between $S L$ and $N L$ obtained from the measured $S N R$ values and equation 9. For most distances at frequencies above $3 \mathrm{Khz}$, the difference of $S L$ and $N L$ is almost the same as the $0 S N R$ plot, and the shape of both plots follows the trend of $T L^{5}$, rising with increasing distance in a logarithmical trend from 0 to $15 \mathrm{~dB}$. For lower frequencies, the gap between the two plots widens, corresponding to a higher $S N R$ and a larger measured value for $S L-N L$.

In addition to profiling the channel frequency response, the results in Figures 4 and 5 also provide insight into the software modem design. Depending on our target $S N R$ ratio for the network, we can determine the number of frequencies that can be used for reliable communication. For instance, for a target $S N R$ of $5 \mathrm{~dB}$, the plot for all distances up to $9 \mathrm{~m}$ have at least 8 frequencies with an $S N R$ of more than $5 \mathrm{~dB}$, so the software modem can use an 8-frequency FSK scheme for data transmission for distances of up to $9 \mathrm{~m}$ without violating the signal quality requirement. This insight has proved valuable for developing the data modulation scheme, on which the next section elaborates.

\section{Underwater Data Transmission}

The sensor nodes in our application send small amounts of data, consisting of sensor readings, once every several minutes. Thus, the data rate requirement of this application is small,

\footnotetext{
${ }^{5}$ When a signal is not received, the $S N R$ is 0 and equation 9 reduces to $S L-N L=T L$.
} 


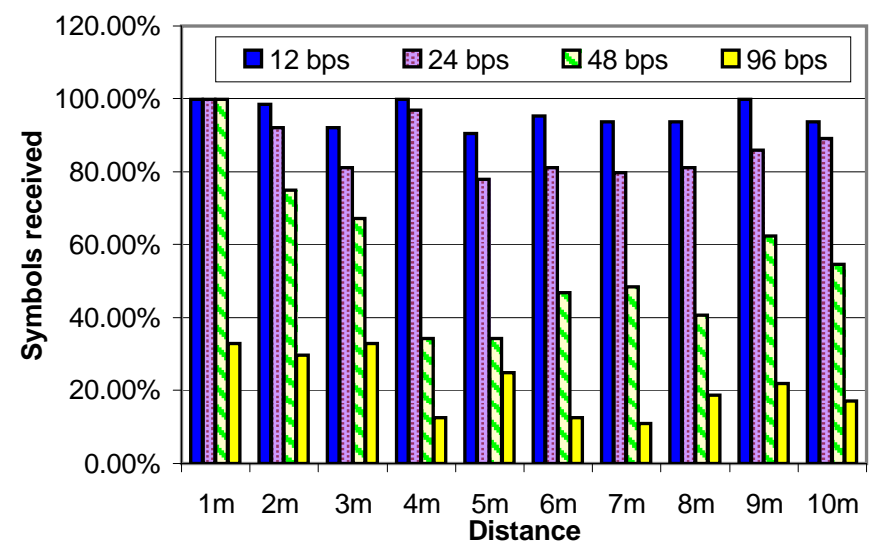

Fig. 6. Data reception capability at different distances and bitrates

so the transmission of tens of bits per second is sufficient. The relaxed requirement on bit rate enables us to maximize the communication range of our network by using only 8 frequencies for FSK modulation. We choose the 8 frequencies with the highest $S N R$ from Figure 4 for our FSK software modem: 400; 500; 600; 700; 800; 900; 1300; and $1400 \mathrm{~Hz}$. Each frequency in our modem encodes 3 bits. To evaluate the impact of the FSK symbol length on the data reception capability at different distance, we consider 4 modems with the following data bit rates: 12; 24; 48 and 96.

Figure 6 plots the percentage of correctly received FSK symbols at different distances and bit rates using the generic hardware. The receiver could successfully receive and demodulate more than $90 \%$ of symbols at 12 bps, and about $80 \%$ at 24 bps. The demodulation capability does not appear to diminish with increased distance for these lower bit rates. For the higher data rates, the receiver could not demodulate a significant portion of the signal beyond $3 \mathrm{~m}$ for $48 \mathrm{bps}$ rates, and at $96 \mathrm{bps}$, the demodulation capability is even lower.

\section{CONCLUSION}

In this paper, we have provided a comparative analysis and experimental study on the channel profile of generic acoustic hardware with software modems for acoustic communications in air and in water. The aerial channel favors frequencies above $1 \mathrm{Khz}$ because of high ambient noise at low frequencies. In contrast, the underwater channel favors lower frequencies as a result of the vinyl membrane for speaker waterproofing which seems to resonate better at lower frequencies. An initial test of the channels's underwater data communication capability has revealed that the channel can transfer data at $12 \mathrm{bps}$ and $24 \mathrm{bps}$, with respective error rates of $10 \%$ and $20 \%$, up to a distance of $10 \mathrm{~m}$. We plan to capitalize on the results obtained here to implement a software acoustic modem on Tmote Invent units and deploy the units in Newport Bay, CA. The longer term field deployment will allow us to better evaluate the acoustic modem in a practical application scenario.

\section{REFERENCES}

[1] C. V. Lopes and P. Aguiar. "Acoustic Modems for Ubiquitous Computing," IEEE Pervasive Computing, Mobile and Ubiquitous Systems, Summer 2003.

[2] R. Jurdak, C. V. Lopes and P. Baldi. Battery Lifetime Estimation and Optimization for Underwater Sensor Networks. Sensor Network Operations, Wiley/IEEE Press. May, 2006 (in press).

[3] MoteIV Corporation www.moteiv.com

[4] Linkquest Inc. available: www.link-quest.com

[5] DSPComm. available: www.dspcomm.com

[6] R. A. Iltis, H. Lee, R. Kastner, D. Doonan, T. Fu, R. Moore and M. Chin. "An Underwater Acoustic Telemetry Modem for Eco-Sensing," In proc. MTS/IEEE Oceans'05, September 2005.

[7] Utility Acoustic Modem available: auvlab.mit.edu

[8] I. Vasilescu, K. Kotay, D. Rus, M. Dunbabin and P. Corke. "Data Collection, Storage, and Retrieval with an Underwater Sensor Network," In Proc. Sensys' 05, San Diego, CA, 2005.

[9] Blauert, Jens (Ed.) Communication Acoustics. Springer, 2005, ISBN: 3-540-22162-X

[10] R. J. Urick. Principles of Underwater Sound. Mcgraw-Hill, 1983.

[11] Sony SRS-P7 PC speakers. available: www.sony.com

[12] N. Fruehauf and J.A. Rice. System design aspects of a steerable directional acoustic communications transducer for autonomous undersea systems. In OCEANS, volume 1, pages 565 -573. IEEE, 2000.

[13] F. H. Fisher and V. P. Simmons. "Sound Absorption in Sea Water," Journal of Acoustical Society of America, 62:558, 1977.

[14] R. Jurdak, C.V. Lopes, and P. Baldi. "An Acoustic Identification Scheme of Location Systems," In Proc. ICPS'04, Beirut, Lebanon. 2004.

[15] M. Stojanovic. Recent advances in high speed underwater acoustic communications. Oceanic Engineering, 21(4):125-36, 1996.

[16] X. Yang et al. Design of a Wireless Sensor Network for Longterm, In-Situ Monitoring of an Aqueous Environment. Sensors, 2:455-472, 2002. 